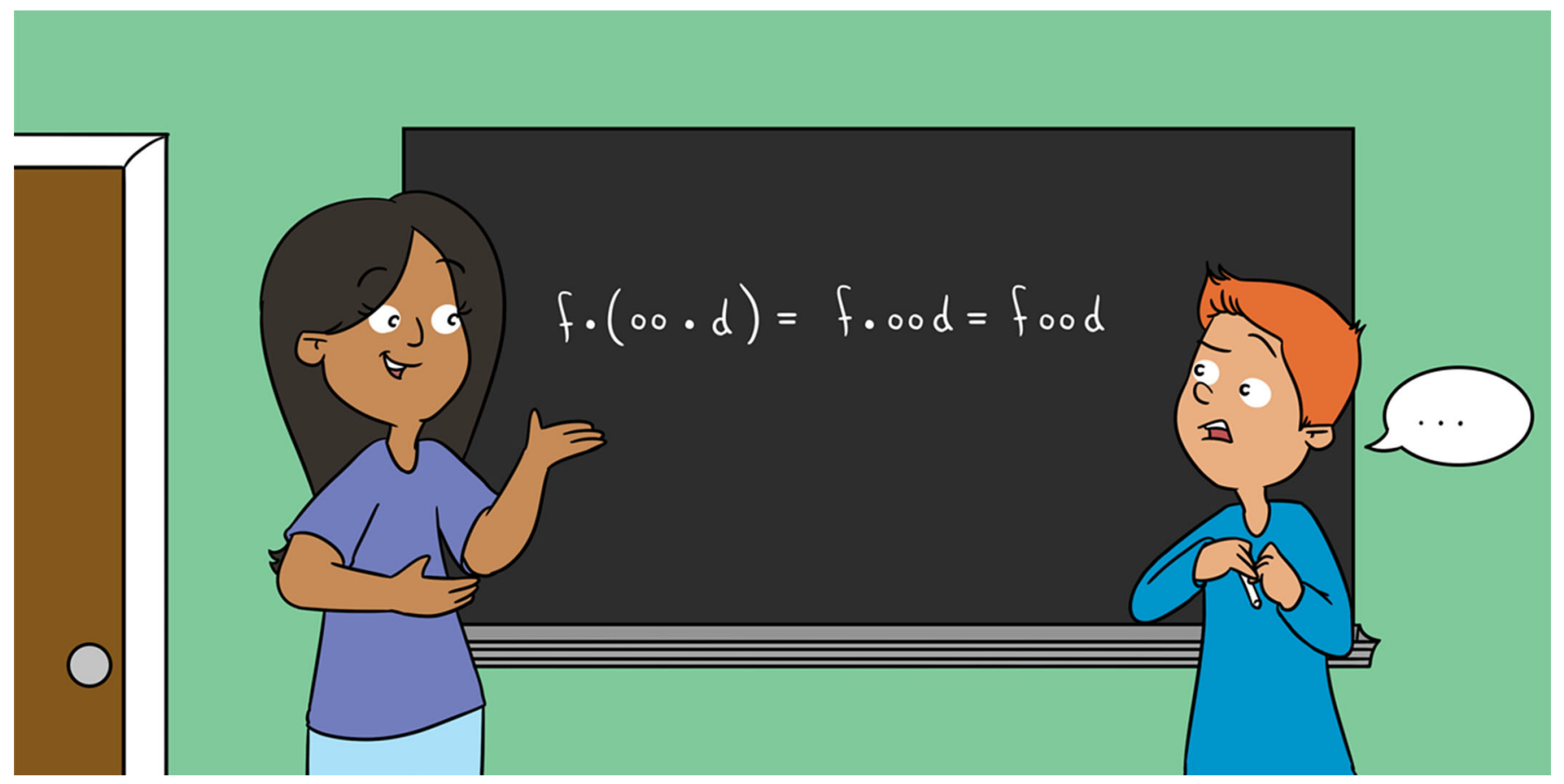

\title{
THE ALGEBRA DETECTIVE: IF SNAPE IS A SNAKE, THEN P = K!
}

\section{Per Bäck ${ }^{1}$ and Gizem Karaali ${ }^{2 *}$}

${ }^{1}$ Division of Mathematics and Physics, The School of Education, Culture and Communication, Mälardalen University, Västerås, Sweden

${ }^{2}$ Department of Mathematics, Pomona College, Claremont, CA, United States

\section{YOUNG REVIEWER:}

GIOVANNI AGE: 10
Algebra is often introduced as arithmetic done with letters. In this article, we follow this analogy and see where it takes us. We ask questions, such as: If "SNAPE = SNAKE," can we conclude that " $\mathrm{P}=\mathrm{K}$ "? Here is the spoiler: the path takes us into what mathematicians call group theory, a subfield of algebra that studies symmetry, and allows us to say interesting things about various languages like English and Swedish (and one very important fact about algebra itself).

\section{WHAT IS ALGEBRA REALLY?}

Everybody is introduced to algebra at some point in school mathematics. Algebra comes to us gradually. First, we solve basic arithmetic problems. For example, we might be offered the expression:

$$
3+5=\square .
$$


We think about our arithmetic facts and fill in the square with 8 . Pretty soon, the square changes places and we see:

$$
3+\square=8 .
$$

We know what is going on, and we say the square has to be 5 this time.

When teachers, textbook writers, and curriculum developers decide we are ready for it, the boxes disappear, getting replaced by letters. So, in a class called Algebra, a student might see:

$$
3+x=8
$$

To solve this problem, one "isolates the variable," applying the same mathematical operation on both sides of the equality sign ("subtract $3 ")$. This might sound fancy, but the problem is actually the very same as the one before it. The box is now a letter; that is the only difference.

In some very basic sense then, school algebra is doing arithmetic with letters. This is actually an incomplete, and somewhat inaccurate, definition of algebra, but it will suffice for now.

\section{IF ALGEBRA IS ABOUT LETTERS, WHAT CAN IT TELL US ABOUT ENGLISH?}

In algebra class, we are soon working with more and more complicated expressions involving variables and numbers together, such as:

$$
x y+10=16
$$

"Isolating the variables" gives us:

$$
x y=6 .
$$

If we are told that $x=3$, we can plug in the value for $x$ and see:

$$
3 y=6
$$

Dividing both sides by 3 yields $y=2$.

Eventually, we get really good at noticing common factors on both sides of the equality sign and canceling them out: $4 x=16$ becomes $x=4,11 y=33$ becomes $y=3$, and so on.

Could we do all of algebra with only letters, the way we do arithmetic with only numbers? In that case, letters could even be actual letters, 
not placeholders. What would it mean to say that two expressions are equal to one another in this scenario? For example, Professor Severus Snape of Hogwarts is a famous Slytherin, so it makes sense to say that "SNAPE = SNAKE." We could also say:

$$
\text { authors of this article }=\text { Per }+ \text { Gizem, }
$$

or,

$$
\text { my favorite food = ice cream. }
$$

This last one is tricky, because "my" is always context dependent. For example, if Gizem is reading this sentence, "my favorite food" will mean "Gizem's favorite food" and the resulting sentence will be a true statement. However, if her sister were reading the same sentence, she would be asserting that Gizem's sister's favorite food is ice cream, which is definitely not a true statement (Yes, there are people who just do not like ice cream. Weird, we know!). One could even consider pronouns to be the ultimate variables. After all, each pronoun takes the place of a noun determined by the context of the earlier conversation.

Another thing we could do using only letters is to make two expressions equal if they sound the same. For example, in English, the letter collection "you" sounds the same as the single letter "u." So, we could set them equal to one another:

$$
\text { you }=\mathrm{u} .
$$

Does your eager algebra eye want to do some cancellations? Do you think you can cancel the "u"s on both sides and get "yo = 1"? Why not? But then "dye = die" gives you " $y=i "$ and so on. How far can you go? And just what does " $y=i$ " mean?

A bag of objects together with an operation that takes two objects from the bag to make another object that is also in the bag. The operation needs to obey the associative law, and the special object theone must be in the bag. Last, all objects in the bag must have an inverse that is also in the bag.

\section{OPERATION}

A way of taking two objects from a bag to form a new object that is also in the bag.

\section{WHAT IS GOING ON? A GROUP THEORY PRIMER}

In mathematics, group theory is the field of algebra that studies symmetry using certain algebraic structures called groups. A group is a bag of objects (see also [1]) together with a way of taking two objects from the bag to make another object that is also in the bag. The objects can be anything: numbers, words, or even ice cream cones. The "way" is called an operation and is often written "•": if object1 and object2 are two objects in the bag,

\section{object1 • object2}

is the object in the bag we make from object1 and object2 with the operation "•." We can form yet another object by first taking the object object $1 \bullet$ object 2 and then the object object 3 from the bag, to get the 


\section{ASSOCIATIVE LAW}

If "•" is an operation and object1, object2, and object 3 are objects in some bag, the associative law says that the object (object1 • object2) • object3 is the same object as object 1 • (object2 • object3).

\section{INVERSE}

If "•" is an operation and object an object in some bag, the inverse of object, let us call it inverseobject, is an object in the bag such that

object $\bullet$ inverseobject $=$ inverseobject $\bullet$ object $=$ theone. Here, theone is the special object in the bag.

object

$$
\text { (object1 • object2) • object3. }
$$

But what if we took instead object1 first and then

$$
\text { object2 • object3? }
$$

In a group, the resulting object is the same! In other words, we require that what is called the associative law holds:

$$
(\text { object1 } \bullet \text { object2 }) \bullet \text { object3 }=\text { object1 } \bullet(\text { object2 } \bullet \text { object3 })
$$

We also require that there is a special object in the bag, let us call it theone, since it is so special, such that for any object object in the bag,

$$
\text { object } \bullet \text { theone }=\text { theone } \bullet \text { object }=\text { object. }
$$

Our last requirement is that we should be able to cancel objects on both sides of "=" to get the resulting object theone. This can be expressed as follows: we want any object object in the bag to have a buddy in the bag called an inverse, say inverseobject, such that

$$
\text { object } \bullet \text { inverseobject }=\text { inverseobject } \bullet \text { object }=\text { theone } .
$$

That's it! A bag of objects with an operation that satisfies these three requirements is called a group.

Example 1: We could take the bag of objects to be all the numbers (positive, negative, and zero) and "•" to be "+." For example, $3+4=7$ and $7+2=9$, so $(3+4)+2=7+2=9$. We also have $4+2=6$ and $3+6=9$, so $3+(4+2)=3+6=9$. In other words, $(3+4)+2=3+(4+2)$. This holds true for all numbers in our bag. Our special object theone turns out to be the number 0 . Since $7+(-7)=0$, the inverse of 7 has to be -7 . Can you figure out what the inverse of any number must be?

Example 2: Let us try to make a group out of the letter $x$. We start by putting $x$ in our bag of objects. In order to make this a group, we also need to add the special object theone to our bag. Instead of writing theone, which is a bit cumbersome, we use the symbol 1. Last, we also need to add the inverse of $x$. We put a hat on $x$ and simply say that this new letter, $\widehat{x}$, is the inverse of $x$. This means that with $x, \widehat{x}$, and 1 in our bag, $x \bullet \widehat{x}=1$. Is this a group? No, not yet. For example, $x \bullet x$ also needs to be in our bag, so we simply add it and its inverse $\widehat{x} \bullet \widehat{x}$ as well. Continuing like this and assuming that the associative law holds, we get the so-called group generated by $x$.

Example 3: Suppose that we take the group generated by $x$ as described in Example 2 and add the rule $x=1$. If we replace $x$ by 1 


\section{TRIVIAL GROUP}

The smallest group possible, the bag of objects containing only one object, theone. in $x \bullet \widehat{x}=1$, we get $1 \bullet \widehat{x}=\widehat{x}=1$. We also get $x \bullet x=1 \bullet 1=1$. In fact, all objects in our bag must be equal to 1 . In other words, our bag contains only one object, 1. From the definition, the bag in a group needs to have at least one object in it, theone. Therefore, this must be the smallest group possible, so it is called the trivial group.

Example 4 | Let us explore a more visual example. Consider the Scrabble tile for the letter $R$ :

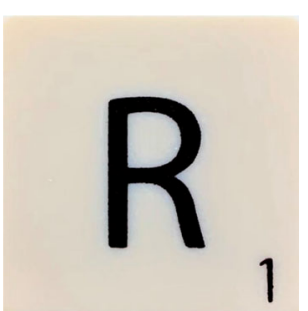

Photo: Per Bäck.

This tile can be rotated $90^{\circ}$ clockwise to give us

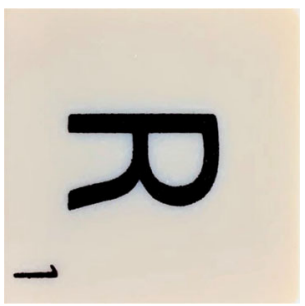

Photo: Per Bäck.

If you rotate it $90^{\circ}$ more, you get:

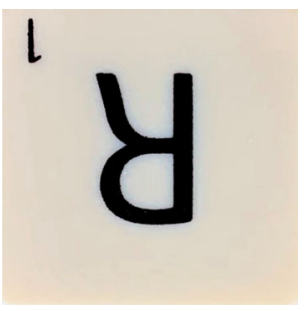

Photo: Per Bäck.

Another $90^{\circ}$ rotation gives you:

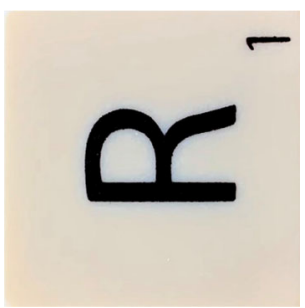

Photo: Per Bäck.

and a final $90^{\circ}$ rotation will get you back to the original tile. This is a nice example of a group. Each of these operations can be reconstructed from the single clockwise rotation by $90^{\circ}$. If we set $x$ to be the "rotate clockwise by $90^{\circ}$ operation, then the group we get is the bag containing $x, x^{2}, x^{3}$, and $x^{4}$; this last one is the same as theone, or in this context, the "do-nothing" operation. Do you see what the inverses are? 


\section{HOMOPHONIC GROUPS}

Can we make a group out of the whole alphabet? The English alphabet consists of 26 letters $a, b, c, \ldots, z$. We invent the inverse English alphabet to be the letters of the English alphabet with hats on them, $\widehat{a}, \widehat{b}, \widehat{c}, \ldots, \widehat{z}$. We form all possible words from the letters in the two alphabets and put them in a bag. By a word, we mean any combination of letters, so qwertyasdf, brain, $d$, and foo are all words in our bag. Whenever a letter lies immediately next to its inverse letter in a word, we cancel the two letters. This means that

$$
\text { foo }=f
$$

and

$$
\text { yoûu }=\text { yo. }
$$

We also invent an operation $\bullet$ on the bag of words to be that of gluing two words together to make a new word, so that

$$
d \bullet f \circ o=d f \circ o
$$

and

$$
\text { foo } \bullet d=\text { food }
$$

This also means that

$$
a \cdot \widehat{a}=a \widehat{a}=,
$$

\section{EMPTY WORD}

The word with no letters in it. the word consisting of no letters at all-the empty word! Continuing, the operation • obeys the associative law. For example,

$$
f \bullet(\circ o \bullet d)=f \bullet \text { ood }=\text { food }
$$

and

$$
(f \bullet \circ) \bullet d=\text { foo } \bullet d=\text { food } \text {. }
$$

This almost looks like a group where the special object is the empty word! However, we need not only the letters to have inverses, but all the words as well. This works if we say that the inverse of a word is the word spelled backwards and with a hat on each letter. For example, the inverse of yo is $\widehat{o y}$ since then

$$
y o \bullet \widehat{o y}=y o \widehat{o y}=y \widehat{y}=.
$$

Just as in Example 2, we could use the symbol 1 for the special object (the empty word in this case), so that

$$
y \widehat{y}=1
$$




\section{FREE GROUP}

The group made from some alphabet by using the operation of gluing letters together to form words, and then gluing words together to form new words. In the bag of objects are all words possible, including the empty word, which is the special object theone.

HOMOPHONIC GROUP

The free group on some alphabet where words that sound the same are equal.
The above group is called the free group on the English alphabet. Free, because it is the freest way one can make a group out of the English alphabet.

Now, suppose that we add the rule that two words are equal if they sound the same, as we suggested at the end of the second section. Then this means that

$$
\text { you }=u \text {. }
$$

We can cancel the " $u$ "s by using the operation $\bullet$ together with $\widehat{u}$ on each side. On the left-hand side, we get

$$
\text { you } \bullet \widehat{u}=y o u \widehat{u}=y o,
$$

and on the right-hand side

$$
u \bullet \widehat{u}=u \widehat{u}=1
$$

so yo $=1$

Words that sound the same are called homophones, and the free group on the English alphabet where homophones are equal is called the homophonic group of English. The word you and the letter $u$ are therefore the same in the homophonic group of English, and by the above calculations, so are yo and the empty word (for which we used the symbol 1). Mathematicians have shown that the homophonic groups of English and French are the groups with just the empty word in them-the trivial group [2]. Other authors have shown that the homophonic group of German is the trivial group, while those of Korean and Turkish are not [3].

\section{SWEDISH, ANYONE?}

Why not learn Swedish while doing group theory? In fact, we will see that it is trivial! The Swedish alphabet has 29 letters in it: the 26 letters that form the English alphabet together with the three funny-looking letters å, ä, and ö. By using a standard dictionary like [4], we will see that the homophonic group of the Swedish language is the trivial group. First, we use something that cannot be found in the Swedish Academy's dictionary [4], but is well-known to all Swedish mathematicians, namely that om and omm are pronounced the same. In other words,

$$
\mathrm{om}=\mathrm{omm}
$$

so by canceling om on both sides, $m=1$. Continuing,

$$
t=t e, \quad j u l=h j u l, \quad \text { värd }=\text { värld }, \quad \text { an }=a n n, \quad \text { pol }=p o o l \text {, }
$$


so $e=h=l=n=0=1$. We also have

upplåst $=$ uppblåst,$\quad$ hårdra $=$ hårddra,$\quad$ bygd $=$ byggd , saltare $=$ psaltare, $\quad$ avisa $=$ avvisa

which gives us $b=d=g=p=v=1$. Since

$$
\text { alf }=\text { alv, } \quad \text { jäst }=g a ̈ s t, \quad h a ̊ v=h o v, \quad b a ̈ c k=b e c k,
$$

we have $f=v=1, j=g=1, \stackrel{a}{=}=0=1, \ddot{a}=e=1$. Now,

$$
\text { board }=\text { bård }, \quad \text { sig }=\text { sej, } \quad \text { skälva }=\text { själva }, \quad r=\text { ärr } \text {, }
$$

and so $a=1 a=o a=a ̊ n=1, i=i 1=i g=e j=1, k=j=1$, and $r=1 r r$ gives us $r=1$. Moreover,

$$
\text { chippa }=\text { kippa }, \quad \text { wienare }=\text { vinare }, \quad \text { gray }=\text { grej, }
$$

so we must have $c=c 1=c h=k=1, w=w 1=w i e=v i=1$, and $y=1 y=a y=e j=1$. Now,

$$
\text { sensor }=\text { censor }, \quad \text { tjeck }=\text { check, }
$$

and so $s=c=1, t=t 1=t j=c h=1$. We have

$$
\text { koxa }=k o k s a, \quad z o o=s o,
$$

which gives us $x=k s=1, z=z 1=z 0=s=1$. We also have

$$
\operatorname{sioux}=\text { so, }
$$

so we get $u=1 u 1=\operatorname{sioux}=$ so $=1$. At last,

$$
\text { toquen = tocken, övre = oeuvre, }
$$

and therefore $q=q 1=q u=c k=1$ and $\ddot{o}=o e u=1$. We have shown that all the 29 letters must be equal to 1 . Since the only word we can form from 1 is 1, we have shown that the homophonic group of Swedish is trivial!

\section{SO WHAT?}

Why is all of this important? Here is an argument that might appeal to many mathy folks: Since any word in the trivial group is the empty word 1 and English is trivial, algebra $=1$ and awesome $=1$. Therefore, algebra = awesome. Now isn't that something?

On a more serious note, the homophonic group of a language tells us how well the alphabet represents what the language sounds like. 
English, French, German, and Swedish, whose homophonic groups are all trivial, have many words where different letters are pronounced the same. Korean and Turkish, whose homophonic groups are non-trivial, have younger alphabets whose letters sound more similar to what they do in actual words. In other words, just playing around with letters in fun and seemingly meaningless ways can actually tell us something interesting about languages and their writing systems. Maybe the next time someone asks you what algebra is good for, you can tell them that it can help us understand something as human as language!

If you are excited by this article and want to learn more about groups, there are many books out there that might be good next steps. Alternatively, you might check out this interesting article on more examples of groups [5], or this neat online exploration of one of the most ambitious group theory projects of the twentieth century [6].

\section{REFERENCES}

1. Bouyer, F. 2018. Infinity and trying to do maths with it. Front. Young Minds 6:61. doi: $10.3389 /$ frym.2018.00061

2. Mestre, J. F., Schoof, R., Washington, L., and Zagier, D. 1993. Homophonic quotients of free groups [Quotients homophones des groupes libres]. Exp. Math. 2:153-5. doi: 10.1080/10586458.1993.10504275

3. Gangl, H., Karaali, G., and Lee, W. 2019. Homophonic quotients of linguistic free groups: German, Korean, and Turkish. Involve 12:463-74. doi: 10.2140/ involve.2019.12.463

4. The Swedish Academy. 2020. Svenska Akademiens Ordböcker. Available online at: https://svenska.se/ (accessed October 20, 2020).

5. Roney-Dougal, C. 2006. The Power of Groups. Plus Magazine. Available online at: https://plus.maths.org/content/os/issue39/features/colva/index.

6. Elwes, R. 2006. An Enormous Theorem: The Classification of Finite Simple Groups. Plus Magazine. Available online at: https://plus.maths.org/content/ os/issue41/features/elwes/index

SUBMITTED: 01 January 2020; ACCEPTED: 02 November 2020; PUBLISHED ONLINE: 02 December 2020.

EDITED BY: Marco Aldi, Virginia Commonwealth University, United States

CITATION: Bäck P and Karaali G (2020) The Algebra Detective: If Snape Is a Snake, Then $\mathrm{P}=\mathrm{K}$ ! Front. Young Minds 8:524026. doi: 10.3389/frym.2020.524026

CONFLICT OF INTEREST: The authors declare that the research was conducted in the absence of any commercial or financial relationships that could be construed as a potential conflict of interest.

COPYRIGHT @ 2020 Bäck and Karaali. This is an open-access article distributed under the terms of the Creative Commons Attribution License (CC BY). The use, 




distribution or reproduction in other forums is permitted, provided the original author(s) and the copyright owner(s) are credited and that the original publication in this journal is cited, in accordance with accepted academic practice. No use, distribution or reproduction is permitted which does not comply with these terms.

\section{YOUNG REVIEWER}

\section{GIOVANNI, AGE: 10}

I was born in California and then lived in the Southwest for almost half my life, but now I am living in Virginia. I usually play videogames at home but I also do other things. I really like art and sketching. I won the state fair art contest. I want to be an architect or engineer when I grow up. I also do like writing stories, I am working on a story or book called "Within Our Walls."

\section{AUTHORS}

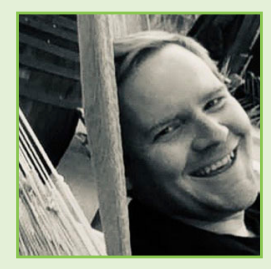

\section{PER BÄCK}

Per is a Ph.D. student in algebra at Mälardalen University in Sweden. His research is mainly focused on non-associative and non-commutative algebra. He does not like pancakes.

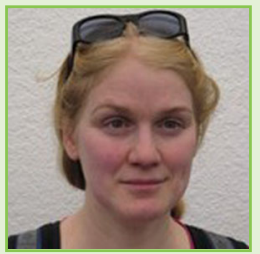

\section{GIZEM KARAALI}

Gizem is a Professor of Mathematics at Pomona College in California, USA. Groups are ubiquitous in her research in representation theory and algebraic combinatorics. Gizem is an unrepentant chocoholic. *gizem.karaaliapomona.edu 